\title{
Exceptions that prove the rule
}

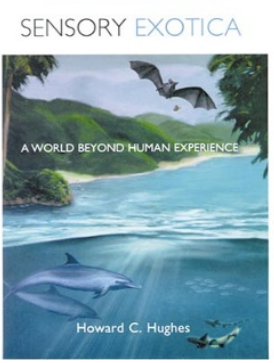

\section{Sensory Exotica: A World Beyond Human Experience}

\author{
by Howard Hughes \\ MIT Press, 1999. $\$ 26.95$ \\ hardcover, pp 344 \\ ISBN 0-262-08279-9
}

Reviewed by Harold H. Zakon

Some animals live in sensory worlds that are unimaginable to us. Bats and dolphins, which operate on fast-forward, construct complex neural images of the environments through which they hurtle by information encoded in the reflections of ultrasonic 'sound bites.' Electric fish probe their surroundings with brief electrical pulses and sense nearby objects by the microsecond shifts in timing and microvolt changes in amplitude these objects cause in their self-generated electric fields. Some electric fish 'digitize' information at frequencies exceeding a kilohertz with submicrosecond jitter. These are feats that push the envelope of ion channel behavior and neural processing.

Bees forage on cloudy days by detecting complex patterns of polarized light in the sky. This is done with a small dorsal patch of retina comprising a few hundred specialized ommatidia. The exact positioning of a single ultraviolet-sensitive photoreceptor within each ommatidium, and the variation of its position within the ommatidia across the dorsal patch, allows a bee to perceive the brightest signal when it is lined up with and flying towards the unobservable sun. This is a simple but elegant solution by strategic positioning of an array of detectors, a so-called 'computational anatomical' solution.

It is awe-inspiring to consider how animals accomplish these feats; it is no less so to trace the paths of discovery leading from our cloistered anthropocentrism to the realization that many animals operate in a realm unknowable to us. Sensory Exotica: A World Beyond Human Experience attempts to peer into these private worlds and inspire us in both ways.

Harold Zakon is in the Section of Neurobiology $\&$ Institute for Neuroscience, University of

Texas, Austin, Texas 78712, USA.

e-mail:h.zakon@mail.utexas.edu
This book, which grew out of an undergraduate course taught by the author, is not a textbook but a popular science book. It is at the level of a companion book for an introductory collegelevel course in sensory biology or perceptual psychology, or for the interested non-scientist. It is generally well crafted, clearly written and engaging. The book includes sections on echolocation by bats and dolphins, electroreception by electric fish, magnetoreception in a variety of animals, ultraviolet light and polarized light reception in bees and ants, and pheromone reception in mammals and insects. Included in this last section is an overview of the evidence for the sensitivity of our own olfactory vomeronasal organs to putative human pheromones. This section is intriguing because it emphasizes the surprising fact that we ourselves possess sensory capacities that are beyond our direct perception.

Although this book is aimed at a non-technical audience, the author does not shrink from explaining some fairly technical points. His explanations of mathematical or physical principles such as Fourier analysis, vector addition, the electromagnetic spectrum and polarization of light are lucid and vivid. His descriptions of complex biological phenomena such as Doppler shift compensation in bats, the jamming avoidance response of electric fish, or the relationship between photopigment orientation, photoreceptor organization and polarized light perception are cogent and approachable. Moreover, he does this without getting bogged down in detail or lessening the reader's sense of wonder for the abilities of these animals and the mechanics of their sensory systems.

With a few exceptions, such as a description of recent experiments identifying a plausible magnetoreceptor, most of the work cited in this book is classical; this is not a volume for those interested in the cutting edge. Rather, the author dwells on the history of discovery, the nature of scientific investigation and the sociology of science in these fields. He describes how these unusual senses were first discovered and applauds the simple but clever behavioral experiments used to glimpse into an animal's mind and comprehend what we cannot directly perceive. He illustrates for each unusual sensory system the movement from initial skeptical dismissal by fellow scientists to final acceptance by the weight of internally consistent, reproducible evidence. In many ways, the sociohistorical aspects of doing science are as much the main message of this book as the sensory biology of these wonderful animals.

I found this book to be an enjoyable read, although it has its share of minor annoyances. The author occasionally walks a fine line between enhancing the science and distracting the reader with anecdotes and asides. The tone of the book is chatty in places, and the quality of some of the figures is pixilated and amateurish. There are the occasional factual errors (at least in electroreception, my area of expertise). These errors, however, would likely only bother an expert and do not detract from the main points.

As a neurobiologist, I found Sensory Exotica a regrettable choice for a title because it reinforces the notion of these animals as unusual, albeit intriguing. Luckily, in a final epilogue, the author reunites these separate islands into the larger Gondwanaland of neurobiology to show how they are the exceptions that prove the rule. He correctly emphasizes that although these animals may have fancy transducers and high-end neural processors not possessed by less-blessed animals, in a fundamental sense they are no different from anybody else. Rather than being oddball, their sensory systems are showcases of the exquisite development of the normal. Such sensory systems are not only understandable in terms of common neurobiological principles, but they illuminate them.

The world at large envisions scientists as detectives. Burdened by grant writing and other daily chores, we often forget that vision. This book will rekindle that notion, hopefully even in the jaded professional. I recommend this book highly to its intended audience as well as to the practicing scientist eager to read a wellpaced and relaxing book. 